\title{
The structure of the magnetic field in the massive star-forming region $\mathrm{W} 75 \mathrm{~N}$
}

\author{
G. Surcis $^{1, \star}$, W. H. T. Vlemmings ${ }^{1}$, S. Curiel $^{2}$, B. Hutawarakorn Kramer $^{3,4}$, J. M. Torrelles ${ }^{5}$, and A. P. Sarma ${ }^{6}$ \\ 1 Argelander-Institut für Astronomie der Universität Bonn, Auf dem Hügel 71, 53121 Bonn, Germany \\ e-mail: gsurcis@astro.uni-bonn.de \\ 2 Instituto de Astronomía (UNA), Apdo Postal 70-264, Cd. Universitaria, 04510-Mexico DF, Mexico \\ 3 Max-Planck Institut für Radioastronomie, Auf dem Hügel 69, 53121 Bonn, Germany \\ ${ }^{4}$ National Astronomical Research Institute of Thailand, Ministry of Science and Technology, Rama VI Rd., Bangkok 10400, Thailand \\ 5 Instituto de Ciencias del Espacio (CSIC)-UB/IEEC, Universitat de Barcelona, Martí i Franquès 1, 08028 Barcelona, Spain \\ ${ }^{6}$ Physics Department, DePaul University, 2219 N. Kenmore Ave., Byrne Hall 211, Chicago, IL 60614, USA
}

Received 27 September 2010 / Accepted 16 November 2010

\begin{abstract}
Context. A debated topic in star formation theory is the role of magnetic fields during the protostellar phase of high-mass stars. It is still unclear how magnetic fields influence the formation and dynamics of massive disks and outflows. Most current information on magnetic fields close to high-mass protostars comes from polarized maser emissions, which allows us to investigate the magnetic field on small scales by using very long-baseline interferometry.

Aims. The massive star-forming region W75N contains three radio continuum sources (VLA 1, VLA 2, and VLA 3), at three different evolutionary stages, and associated masers, while a large-scale molecular bipolar outflow is also present. Very recently, polarization observations of the $6.7 \mathrm{GHz}$ methanol masers at milliarsecond resolution have been able to probe the strength and structure of the magnetic field over more than 2000 AU around VLA 1. The magnetic field is parallel to the outflow, suggesting that VLA 1 is its powering source. The observations of $\mathrm{H}_{2} \mathrm{O}$ masers at $22 \mathrm{GHz}$ can give more information about the gas dynamics and the magnetic fields around VLA 1 and VLA 2.

Methods. The NRAO Very Long Baseline Array was used to measure the linear polarization and the Zeeman-splitting of the $22 \mathrm{GHz}$ water masers in the star-forming region $\mathrm{W} 75 \mathrm{~N}$.

Results. We detected 124 water masers, 36 around VLA 1 and 88 around VLA 2 of W75N, which indicate two different physical environments around the two sources, where VLA 1 is in a more evolved state. The linear polarization of the masers confirms the tightly ordered magnetic field around VLA 1, which is aligned with the large-scale molecular outflow, and also reveals an ordered magnetic field around VLA2, which is not parallel to the outflow. The Zeeman-splitting measured on 20 of the masers indicates strong magnetic fields around both sources (the averaged values are $\left|B_{\mathrm{VLA} 1}\right| \sim 700 \mathrm{mG}$ and $\left|B_{\mathrm{VLA} 2}\right| \sim 1700 \mathrm{mG}$ ). The high values of the magnetic field strengths, which come from the shock compression of the gas, are consistent with the methanol and $\mathrm{OH}$ magnetic field strengths. Moreover, by studying the maser properties we were also able to determine that the water masers are pumped in C-shocks in both sources.
\end{abstract}

Key words. stars: formation - masers - polarization - magnetic fields - ISM: individual objects: W75N

\section{Introduction}

During the formation of low-mass stars, the magnetic field is thought to slow the collapse, to transfer the angular momentum, and to power the outflow, but its role during the formation of high-mass stars is still under debate (e.g., McKee \& Ostriker 2007; Girart et al. 2009). Several questions remain unanswered because massive star-forming regions are rare and distant and often consist of a large number of protostars, consequently it is difficult to observe magnetic fields during their protostellar phase. Recently some progress has been made. Vlemmings et al. (2010) and Surcis et al. (2009, hereafter S09) have shown that the magnetic field is orthogonal to large rotating disks and parallel to molecular bipolar outflows even during the high-mass protostellar phase and not only during the formation of low-mass stars (e.g., Matsumoto \& Tomisaka 2004; McKee \& Ostriker 2007).

* Member of the International Max Planck Research School (IMPRS) for Astronomy and Astrophysics at the Universities of Bonn and Cologne.
They obtained their results by using methanol masers as probes of magnetic fields. In fact, the bright and narrow spectral line emission of masers is ideal for detecting, with polarimetric interferometry, both Zeeman-splitting and the orientation of the magnetic field on scales from arcsec to milliarcsec (mas). So far, the main maser species used for this purpose are $\mathrm{H}_{2} \mathrm{O}, \mathrm{OH}$, and $\mathrm{CH}_{3} \mathrm{OH}$ masers. Since their masing conditions are different they are located in distinct zones of massive star-forming regions. The $\mathrm{H}_{2} \mathrm{O}$ masers are detected in the denser zones with hydrogen number densities $n_{\mathrm{H}_{2}}$ between approximately $10^{8}$ and $10^{10} \mathrm{~cm}^{-3}$ (Elitzur et al. 1989), showing a velocity width of about $1 \mathrm{~km} \mathrm{~s}^{-1}$ and a high brightness temperature $T_{\mathrm{b}}>10^{9} \mathrm{~K}$ (e.g., Reid \& Moran 1981). The first very long baseline interferometry (VLBI) observations of the linear and circular polarization of water maser emissions were made by Leppänen et al. (1998) and Sarma et al. (2001), respectively. Afterwards more $\mathrm{H}_{2} \mathrm{O}$ maser polarization observations were carried out by various authors, which confirmed the importance of this kind of observation in studying the role of the magnetic field in massive star 
formation (e.g., Imai et al. 2003; Vlemmings et al. 2006). Here we present Very Long Baseline Array (VLBA) linear and circular polarization observations of water masers in the high-mass star-forming region $\mathrm{W} 75 \mathrm{~N}$.

$\mathrm{W} 75 \mathrm{~N}$ is an active high-mass star-forming region in the molecular complex DR21-W75 (Dickel et al. 1978; Persi et al. 2006) at a distance of $2 \mathrm{kpc}$. At the resolution of $\sim 1^{\prime \prime} .5$ three $\mathrm{H}_{\text {II }}$ regions were identified (Haschick et al. 1981): W75N (A), $\mathrm{W} 75 \mathrm{~N}(\mathrm{~B})$, and $\mathrm{W} 75 \mathrm{~N}(\mathrm{C})$. At 0.'5 resolution, Hunter et al. (1994) detected three subregions in W75N (B): Ba, Bb and Bc. At higher angular resolution ( 0.'1), Torrelles et al. (1997) resolved the subregions $\mathrm{Ba}$ and $\mathrm{Bb}$ further (renaming them VLA 1 and VLA 3), and imaged a third weaker and more compact $\mathrm{H}_{\text {II }}$ region between them, which they named VLA2. A large-scale high-velocity outflow, with an extension greater than $3 \mathrm{pc}$ and a total molecular mass greater than $255 M_{\odot}$, was also detected from W75N (B) (e.g., Shepherd et al. 2003). Shepherd et al. (2003) proposed a multi-outflow scenario where VLA 2 may drive the large-scale outflow, and VLA 1 and VLA 3 are the centers of two other small flows. So far, it has been impossible to determine the main powering source of the $3 \mathrm{pc}$ outflow. Several authors have suggested VLA 1 as the powering source (e.g., Torrelles et al. 1997, S09 and references therein).

Several maser species $\left(\mathrm{H}_{2} \mathrm{O}, \mathrm{OH}\right.$, and $\left.\mathrm{CH}_{3} \mathrm{OH}\right)$ have been detected in W75N (e.g., Torrelles et al. 1997; Baart et al 1986, S09), in particular around the two $\mathrm{H}$ in regions VLA 1 and VLA 2. The $\mathrm{H}_{2} \mathrm{O}$ maser emissions associated with VLA 1 are located along a linear structure parallel to its radio jet with a proper motion of about 2 mas/yr, while those associated with VLA 2 show a circular distribution, which is expanding with a velocity of about 5 mas/yr (Torrelles et al. 2003, hereafter T03). Only one $\mathrm{H}_{2} \mathrm{O}$ maser is associated with VLA 3 (T03). Methanol masers were detected in two groups (A and $\mathrm{B} / \mathrm{C}$ ) located northwest and southeast of VLA 1, respectively. Group A is along a linear structure and group B/C show an arc-like structure (S09). No $\mathrm{CH}_{3} \mathrm{OH}$ masers are associated with VLA 2 (e.g., Minier et al. 2000, S09). However, VLA 2 is the place where the most intensive OH flare took place (Alakov et al. 2005; Slysh et al. 2010). Other $\mathrm{OH}$ maser emission sites are situated on a ring structure around all three radio sources (Hutawarakorn et al. 2002). Based on the different activity in $\mathrm{H}_{2} \mathrm{O}$ and $\mathrm{OH}$ masers, Torrelles et al. (1997) suggest that these three sources are at different evolutionary stages, in particular VLA 1 is the "oldest" source of this region and VLA 3 the "youngest" one (driving a radio HerbigHaro object, Carrasco-González et al. 2010). In this evolutionary scenario, VLA 2 is at an intermediate stage.

In order to determine and investigate the magnetic field of W75N(B), polarization observations of $\mathrm{OH}$ and $\mathrm{CH}_{3} \mathrm{OH}$ maser emissions have been made (e.g., Hutawarakorn et al. 2002; Fish \& Reid 2007; S09). The magnetic field strength obtained from the $\mathrm{OH}$ maser polarization observations was $\sim 7 \mathrm{mG}$ (e.g., Hutawarakorn et al. 2002; Slysh et al. 2002). During the OH maser flare near VLA 2, Slysh \& Migenes (2006) detected a strong magnetic field of more than $40 \mathrm{mG}$ in several maser spots, which increased in the next observations up to $70 \mathrm{mG}$ (Slysh et al. 2010). S09 measured a magnetic field strength of $50 \mathrm{mG}$ around VLA 1 by studying the circular polarized emissions of methanol masers. Hutawarakorn \& Cohen (1996) and S09 investigated the linear polarization of $\mathrm{OH}$ and $\mathrm{CH}_{3} \mathrm{OH}$ masers, respectively, and both suggested that the magnetic field is oriented along the outflow. Although the $\mathrm{OH}$ masers at 1.6 and $1.7 \mathrm{GHz}$ are very susceptible to both internal and external Faraday rotation, at $6.7 \mathrm{GHz}$ the Faraday rotation is only about $5^{\circ}$ indicating that the finding of the large-scale magnetic field aligned with the outflow is robust.

Since the water masers, unlike the $\mathrm{CH}_{3} \mathrm{OH}$ and $\mathrm{OH}$ masers, are found in denser zones of the star-forming regions, it is crucial to study their polarization emission on a small scale. In fact, because they arise close to both radio sources they can give us new information on the role of the magnetic field during the massive star formation, in particular at two different evolutionary stages.

\section{Observations and data reduction}

We observed the massive star-forming region $\mathrm{W} 75 \mathrm{~N}(\mathrm{~B})$ in the $6_{16}-5_{23}$ transition of $\mathrm{H}_{2} \mathrm{O}$ (rest frequency: $22.23508 \mathrm{GHz}$ ) with the $\mathrm{NRAO}^{1}$ VLBA on November 21st 2005. The observations were made in full polarization spectral mode using 4 overlapped baseband filters of $1 \mathrm{MHz}$ in order to cover a total velocity range of $\approx 50 \mathrm{~km} \mathrm{~s}^{-1}$. Two correlations were performed. One with 128 channels in order to generate all 4 polarization combinations (RR, LL, RL, LR) with a spectral resolution of $7.8 \mathrm{kHz}\left(0.1 \mathrm{~km} \mathrm{~s}^{-1}\right)$. The other one with high spectral resolution (512 channels; $1.96 \mathrm{kHz}=0.027 \mathrm{~km} \mathrm{~s}^{-1}$ ), which only contains the circular polarization combinations (LL, RR), to be able to detect Zeeman splitting of the $\mathrm{H}_{2} \mathrm{O}$ maser across the entire velocity range. Including the overheads, the total observation time was $8 \mathrm{~h}$.

The data were reduced using the Astronomical Image Processing Software package (AIPS) following the method of Kemball et al. (1995). The bandpass, the delay, the phase and the polarization calibration were performed on the calibrator J2202+4216, which has been used successfully by Vlemmings et al. (2006) for $\mathrm{H}_{2} \mathrm{O}$ maser polarization observations of Cepheus A. The fringe-fitting and the self-calibration were performed on one of the brightest maser features VLA 1.14 (Table 1). All calibration steps were initially performed on the dataset with modest spectral resolution after which the solutions, with the exception of the bandpass solutions that were obtained separately, were copied and applied to the high spectral resolution dataset. Stokes $I\left(\mathrm{rms}=7.3 \mathrm{mJybeam}^{-1}\right), Q$ $\left(\mathrm{rms}=6.5 \mathrm{mJy} \mathrm{beam}^{-1}\right)$ and $U\left(\mathrm{rms}=6.5 \mathrm{mJy} \mathrm{beam}^{-1}\right)$ data cubes were created using the AIPS task IMAGR (beam-size 2.0 mas $\times 0.7$ mas) from the modest spectral resolution dataset, while the $I$ and $V\left(\mathrm{rms}=10 \mathrm{mJy}^{-1}\right.$ beam $\left.^{-1}\right)$ cubes were imaged from the high spectral resolution dataset and for the same fields. The $Q$ and $U$ cubes were combined to produce cubes of polarized intensity and polarization angle. Since these observations were obtained between two VLA polarization calibration observations $^{2}$, during which the linear polarization angle of J2202+4216 was constant at 13.5 , we were able to estimate the polarization angles with a systemic error of no more than $\sim 2^{\circ}$. The $I$ and $V$ cubes at high spectral resolution were used to determine the Zeeman splitting.

\section{Analysis}

\subsection{Maser identification}

To identify the water maser features in $\mathrm{W} 75 \mathrm{~N}(\mathrm{~B})$ region, we used an identification process divided into three steps: 1) we used a program called "maser finder" (Curiel, pers. comm.),

\footnotetext{
1 The National Radio Astronomy Observatory (NRAO) is a facility of the National Science Foundation operated under cooperative agreement by Associated Universities, Inc.

2 http://www.aoc.nrao.edu/astro/calib/polar
} 
Table 1. All $22 \mathrm{GHz}$ water maser features in $\mathrm{W} 75 \mathrm{~N}$ for which linear and/or circular polarization was detected.

\begin{tabular}{|c|c|c|c|c|c|c|c|c|c|c|c|c|c|}
\hline $\begin{array}{c}\text { (1) } \\
\text { Maser }\end{array}$ & $\begin{array}{l}\text { (2) } \\
\text { group }^{a}\end{array}$ & $\begin{array}{c}\text { (3) } \\
\text { RA } \\
\text { offset } \\
\text { (mas) } \\
\end{array}$ & $\begin{array}{c}\text { (4) } \\
\text { Dec } \\
\text { offset } \\
\text { (mas) }\end{array}$ & $\begin{array}{c}(5) \\
\text { Peak flux } \\
\text { Density(I) } \\
\left(\text { Jy beam }^{-1}\right) \\
\end{array}$ & $\begin{array}{c}(6) \\
V_{\mathrm{LSR}} \\
\left(\mathrm{km} \mathrm{s}^{-1}\right) \\
\end{array}$ & $\begin{array}{c}(7) \\
\Delta v_{\mathrm{L}} \\
\left(\mathrm{km} \mathrm{s}^{-1}\right) \\
\end{array}$ & $\begin{array}{l}(8) \\
P_{1}\end{array}$ & $\begin{array}{l}(9) \\
\chi\end{array}$ & $\begin{array}{c}(10) \\
\Delta V_{\mathrm{i}}^{b} \\
\left(\mathrm{~km} \mathrm{~s}^{-1}\right) \\
\end{array}$ & $\begin{array}{c}(11) \\
T_{\mathrm{b}} \Delta \Omega^{b}\end{array}$ & $\begin{array}{c}(12) \\
P_{\mathrm{V}} \\
\left(\times 10^{-3}\right) \\
\end{array}$ & $\begin{array}{c}(13) \\
B_{\|}\end{array}$ & $\begin{array}{l}(14) \\
\theta^{c}\end{array}$ \\
\hline VLA 1.01 & $\mathrm{C}$ & -95.3612 & -303.062 & $2.76 \pm 0.13$ & 10.7 & 0.38 & $2.3 \pm 0.4$ & $+43.8 \pm 8.3$ & $1.1_{-0.3}^{+0.3}$ & $9.5_{-0.8}^{+0.7}$ & - & - & $79_{-14}^{+10}$ \\
\hline VLA 1.02 & $\mathrm{C}$ & -94.2666 & -300.461 & $3.39 \pm 0.09$ & 10.5 & 0.40 & $3.2 \pm 0.5$ & $+26.4 \pm 9.4$ & $1.1_{-0.2}^{+0.3}$ & $9.6_{-1 .}^{+0.6}$ & - & - & $86_{-13}^{+2}$ \\
\hline VLA 1.03 & $\mathrm{C}$ & -91.6984 & -289.684 & $30.78 \pm 0.19$ & 11.3 & 0.39 & $5.1 \pm 0.8$ & $+29 \pm 1.6$ & $0.8_{-0.3}^{+0.2}$ & $10.3_{-0.3}^{+0.1}$ & 7.8 & $106 \pm 18$ & $74_{-6}^{+13}$ \\
\hline VLA 1.04 & $\mathrm{C}$ & -72.0367 & -217.556 & $9.77 \pm 0.13$ & 10.7 & 0.46 & $3.9 \pm 0.4$ & $-43.0 \pm 1.4$ & $1.5_{-0.4}^{+0.3}$ & $9.7_{-0.3}^{+0.3}$ & 11.3 & $-212 \pm 51$ & $83_{-8}^{+5}$ \\
\hline VLA 1.05 & $\mathrm{C}$ & -71.5315 & -214.531 & $115.41 \pm 0.12$ & 10.6 & 0.44 & $1.7 \pm 0.2$ & $-56.1 \pm 6.5$ & $1.7_{-0.3}^{+0.2}$ & $9.3_{-0.1}^{+0.5}$ & 2.9 & $-54 \pm 9$ & $74_{-14}^{+16}$ \\
\hline VLA 1.06 & $\mathrm{C}$ & -71.3210 & -213.371 & $76.79 \pm 0.12$ & 10.8 & 0.46 & $1.5 \pm 0.2$ & $-59.3 \pm 6.0$ & $1.8_{-0.5}^{+0.2}$ & $9.4_{-0.2}^{+0.6}$ & 5.1 & $-115 \pm 18$ & $66_{-39}^{+11}$ \\
\hline VLA 1.07 & $\mathrm{C}$ & -70.4789 & -210.289 & $10.16 \pm 0.12$ & 11.1 & 0.45 & $1.4 \pm 0.6$ & $-53.9 \pm 11.3$ & $1.9_{-0.3}^{+0.2}$ & $9.2_{-2.0}^{+0.6}$ & 28.4 & $599 \pm 100$ & $72_{-47}^{+89}$ \\
\hline VLA 1.08 & $\mathrm{C}$ & -69.5948 & -206.944 & $158.28 \pm 0.20$ & 11.5 & 0.51 & $2.6 \pm 0.1$ & $-73.5 \pm 5.0$ & $1.8_{-0.6}^{+0.2}$ & $9.7_{-0.1}^{+0.5}$ & 2.8 & $65 \pm 10$ & $69_{-5}^{+20}$ \\
\hline VLA 1.09 & $\mathrm{C}$ & -11.7886 & -121.338 & $6.14 \pm 0.06$ & 10.4 & 0.33 & $1.1 \pm 0.5$ & $-87.2 \pm 3.2$ & $1.0_{-0.1}^{+0.0}$ & $9.0_{-2.0}^{+0.1}$ & - & - & $86_{-52}^{+3}$ \\
\hline VLA 1.10 & $\mathrm{C}$ & -11.4097 & -237.705 & $2.07 \pm 0.20$ & 11.4 & 0.51 & $2.5 \pm 0.4$ & $-77.9 \pm 7.2$ & $2.0_{-0.3}^{+0.1}$ & $9.5_{-1.5}^{-0.0}$ & - & - & $86_{-4}^{+4}$ \\
\hline VLA 1.11 & $\mathrm{C}$ & -9.7679 & -119.442 & $0.39 \pm 0.03$ & 10.3 & 0.51 & $2.7 \pm 0.3$ & $-56.1 \pm 14.2$ & $1.0_{-0.2}^{+0.2}$ & $9.5_{-0.5}^{+0.6}$ & - & - & $83_{-9}^{+7}$ \\
\hline VLA $1.12^{d}$ & B & -2.7366 & -15.713 & $1.13 \pm 0.03$ & 12.8 & 0.38 & $19.4 \pm 0.7$ & $-86.2 \pm 0.7$ & $0.7_{-0.1}^{+0.3}$ & $10.5_{-0.5}^{+0.1}$ & - & - & $90_{-2}^{+2}$ \\
\hline VLA $1.13^{d}$ & B & -2.6945 & -14.427 & $0.97 \pm 0.03$ & 12.8 & 0.40 & $12.4 \pm 2.5$ & $-50.0 \pm 1.4$ & $0.8_{-0.5}^{+0.2}$ & $10.1_{-2.5}^{+0.3}$ & - & - & $90_{-10}^{+10}$ \\
\hline VLA $1.14^{d}$ & B & -2.6945 & 1.568 & $80.02 \pm 0.07$ & 12.6 & 0.44 & $13.5 \pm 0.7$ & $-16.9 \pm 0.6$ & $0.6_{-0.2}^{+0.2}$ & $10.6_{-0.4}^{+\overline{0} .1}$ & - & - & $90_{-5}^{+5}$ \\
\hline VLA $1.15^{d}$ & B & -0.5894 & -36.270 & $63.25 \pm 0.17$ & 11.6 & 0.49 & $13.1 \pm 1.9$ & $-90.0 \pm 3.6$ & $0.7_{-0.2}^{+0.8}$ & $10.7_{-0.6}^{+0.1}$ & - & - & $90_{-7}^{+7}$ \\
\hline VLA $1.16^{d}$ & B & -0.4210 & -35.545 & $111.47 \pm 0.20$ & 11.4 & 0.55 & $12.9 \pm 1.2$ & $-66.9 \pm 0.6$ & $0.7_{-0.2}^{+0.7}$ & $10.7_{-0.3}^{+0.4}$ & 11.3 & $-177 \pm 27$ & $90_{-6}^{+6}$ \\
\hline VLA $1.17^{d}$ & B & 0 & 0 & $2.96 \pm 0.06$ & 12.7 & 0.54 & $22.9 \pm 5.1$ & $-73.7 \pm 3.3$ & $1.1^{e .2}$ & $10.4_{-2.3}^{+0.6}$ & 42.2 & $809 \pm 182$ & $90_{-11}^{+11}$ \\
\hline VLA $1.18^{d}$ & B & 8.2520 & 31.166 & $6.17 \pm 0.06$ & 12.4 & 0.52 & $22.0 \pm 5.0$ & $-89.7 \pm 1.2$ & $1.0^{e}$ & $10.3_{-2.3}^{+0.5}$ & - & - & $90_{-12}^{+11}$ \\
\hline VLA $1.19^{d}$ & B & 8.2941 & 33.016 & $11.39 \pm 0.07$ & 12.6 & & $25.7 \pm 3.5$ & $+58.2 \pm 7.7$ & $0.9^{e}$ & $10.5_{-1.1}^{+0.4}$ & 5.9 & $92 \pm 30$ & $\begin{array}{l}-16 \\
90_{-6}^{+6}\end{array}$ \\
\hline VLA 1.20 & $\mathrm{C}$ & 36.2920 & -230.717 & $1.86 \pm 0.02$ & 8.1 & 0.33 & $2.7 \pm 0.3$ & $+47.1 \pm 2.9$ & $0.9_{-0.1}^{+0.2}$ & $9.5_{-0.3}^{+1.3}$ & - & - & $84_{-11}^{+3}$ \\
\hline VLA $1.21^{d}$ & B & 57.6378 & 17.914 & $3.56 \pm 0.15$ & 11.2 & & - & - & - & - & $25.8^{f}$ & $-398 \pm 102^{e}$ & - \\
\hline VLA $1.22^{d}$ & B & 84.3726 & 42.854 & $6.71 \pm 0.05$ & 9.8 & 0.43 & $9 \pm 0.2$ & $-20.6 \pm 6.2$ & $1.6_{-0.3}^{+0.1}$ & $8.9_{-1.3}^{+0.6}$ & - & - & $78_{-33}^{+12}$ \\
\hline VLA 1.23 & A & 295.3883 & 281.223 & $39.41 \pm 0.05$ & 21.1 & 0.8 & $6 \pm 0.1$ & $-30.6 \pm 8.5$ & - & - & - & - & - \\
\hline VLA 1.24 & A & 332.4381 & 316.208 & 04 & 21. & & $9 \pm 0.1$ & $-31.8 \pm 8.3$ & - & - & - & - & - \\
\hline VLA 1.25 & A & 7.5828 & 121.956 & 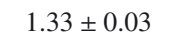 & 23.8 & 0.5 & $3.6 \pm 0.3$ & $-9.9 \pm 7.6$ & $1.5_{-0}^{+0}$ & $7_{-1}^{+0}$ & - & - & $90_{-11}^{+11}$ \\
\hline VLA 2.01 & - & 571.4096 & -488.743 & $1.69 \pm 0.01$ & 5.2 & 0.5 & - & - & - & - & $30.2^{f}$ & $567 \pm 243^{e}$ & - \\
\hline VLA 2.02 & - & 571.9570 & -487.320 & 01 & $5 .($ & 0.58 & - & - & - & - & $21.5^{f}$ & $413 \pm 146^{e}$ & - \\
\hline VLA 2.03 & - & 587.4926 & -621.986 & $12.0 \pm 0.05$ & 6.8 & 0.68 & $5 \pm 0.1$ & $-89.5 \pm 52.7$ & $3.0_{-0.2}^{+0.2}$ & $7_{-0.1}^{+0.5}$ & - & - & $80_{-36}^{+10}$ \\
\hline VLA 2.04 & - & 602.6914 & -611. & 6 & 12.7 & & 0.3 & .8 & - & - & - & - & - \\
\hline VLA 2.05 & - & 604.3755 & -620.571 & $4.62 \pm 0.12$ & 10.9 & 0.50 & $5 \pm 0.4$ & $-80.6 \pm 32.0$ & $1.9_{-0.3}^{+0.2}$ & $9.2_{-2.3}^{+0.1}$ & - & - & $90_{-22}^{+22}$ \\
\hline VLA 1.26 & A & 605.6807 & 212.257 & $20.73 \pm 0.04$ & 18.7 & 0 & \pm 0.1 & $-17.9 \pm 2.0$ & $2.0_{-0.4}^{+0.3}$ & $9.4_{-0.1}^{+0.3}$ & - & - & $85_{-12}^{+3}$ \\
\hline VLA 2.06 & - & 625.2582 & -454.392 & $25.44 \pm 0.16$ & 11.2 & 0.69 & \pm 0.1 & $-47.0 \pm 16.6$ & - & - & - & - & - \\
\hline VLA 2.07 & - & 676.4543 & -432.327 & $1.95 \pm 0.08$ & 6.0 & 0.60 & $2.0 \pm 0.3$ & $+47.7 \pm 8.7$ & $2.3_{-0.2}^{+0.2}$ & $9.4_{-1.9}^{+0.2}$ & - & - & $90_{-15}^{+15}$ \\
\hline VLA 2.08 & - & 677.0438 & -432.545 & $4.65 \pm 0.01$ & 6.2 & 0.59 & $1.1 \pm 0.1$ & $+34.5 \pm 3.1$ & $2.5_{-0.2}^{+0.2}$ & $9.0_{-0.1}^{+0.9}$ & - & - & $83_{-16}^{+8}$ \\
\hline VLA 2.09 & - & 690.0533 & -439.411 & $30.29 \pm 0.06$ & 9.6 & 0.75 & $0.4 \pm 0.1$ & $-58.7 \pm 9.1$ & $3.3_{-0.2}^{+0.1}$ & $8.6_{-0.1}^{+0.1}$ & 3.9 & $-158 \pm 36$ & $76_{-40}^{+10}$ \\
\hline VLA 2.10 & - & 690.7269 & -440.163 & $48.76 \pm 0.06$ & 9.7 & 0.58 & $0.4 \pm 0.1$ & $-69.3 \pm 9.0$ & $2.6_{-0.2}^{+0.1}$ & $8.6_{-0.1}^{+0.1}$ & 6.2 & $-186 \pm 31$ & $72_{-44}^{+8}$ \\
\hline VLA 2.11 & - & 697.1685 & -459.564 & $13.99 \pm 0.04$ & -1.1 & 0.86 & $1.0 \pm 0.3$ & $-39.2 \pm 19.2$ & - & - & - & - & - \\
\hline VLA 2.12 & - & 697.4212 & -460.125 & $3.25 \pm 0.01$ & -2.9 & 2.34 & $1.5 \pm 0.6$ & $-34.2 \pm 12.1$ & - & - & - & - & - \\
\hline VLA 2.13 & - & 697.8843 & -458.710 & $7.16 \pm 0.01$ & 0.5 & 0.69 & $1.0 \pm 0.2$ & $-38.3 \pm 7.5$ & $3.0_{-0.3}^{+0.1}$ & $9.0_{-1.1}^{+0.6}$ & - & - & $82_{-13}^{+9}$ \\
\hline VLA 2.14 & - & 698.6000 & -445.961 & $62.39 \pm 0.07$ & 7.2 & 0.63 & $1.7 \pm 0.2$ & $-84.8 \pm 1.2$ & $2.7_{-0.5}^{+0.3}$ & $9.3_{-0.2}^{+0.7}$ & 1.1 & $-38 \pm 13$ & $74_{-35}^{+15}$ \\
\hline VLA 2.15 & - & 698.9368 & -461.704 & $49.20 \pm 0.05$ & -0.7 & 0.87 & $2.0 \pm 0.2$ & $+78.2 \pm 36.9$ & $3.4^{e}$ & $9.6_{-0.3}^{+0.4}$ & - & - & $68_{-41}^{+3}$ \\
\hline VLA 2.16 & - & 699.3157 & -446.842 & $30.98 \pm 0.05$ & 6.8 & 0.58 & $1.3 \pm 0.2$ & $-75.4 \pm 1.6$ & $2.5_{-0.3}^{+0.2}$ & $9.1_{-0.3}^{+0.7}$ & 15.6 & $470 \pm 73$ & $77_{-33}^{+13}$ \\
\hline VLA 2.17 & - & 701.5051 & -448.017 & $12.73 \pm 0.05$ & 8.3 & 0.53 & $2.5 \pm 0.5$ & $-71.7 \pm 1.7$ & $2.1_{-0.3}^{+0.3}$ & $9.5_{-0.9}^{+0.8}$ & 16.1 & $425 \pm 76$ & $77_{-34}^{+13}$ \\
\hline VLA 2.18 & - & 702.3892 & -449.426 & $4.32 \pm 0.03$ & 9.3 & 0. & $1.2 \pm 0.1$ & $-76.9 \pm 4.4$ & - & - & - & - & - \\
\hline VLA 2.19 & - & 703.4839 & -451.126 & $39.57 \pm 0.17$ & 11.6 & 0. & $2.6 \pm 0.3$ & $-72.6 \pm 3.7$ & - & - & - & - & - \\
\hline VLA 2.20 & - & 703.8628 & -451.496 & $72.56 \pm 0.16$ & 11.2 & 1.09 & $2.0 \pm 0.3$ & $-65.1 \pm 3.9$ & $3.1_{-0.9}^{+0.1}$ & $10.2_{-0.2}^{+0.3}$ & - & - & $61_{-42}^{+42}$ \\
\hline VLA 2.21 & - & 705.5048 & -458.549 & $2.48 \pm 0.01$ & 0.2 & 0.53 & $1.4 \pm 0.3$ & $-8.7 \pm 5.2$ & $2.1_{-0.3}^{+0.1}$ & $9.2_{-2.0}^{+0.2}$ & - & - & $90_{-16}^{+16}$ \\
\hline VLA 2.22 & - & 709.9675 & -458.458 & $4.02 \pm 0.01$ & 5.0 & 0.56 & $0.8 \pm 0.1$ & $-8.0 \pm 4.4$ & & $8.9_{-0.4}^{+0.5}$ & 20.8 & $957 \pm 239$ & $84_{-11}^{+6}$ \\
\hline VLA 2.23 & - & 710.3886 & -459.141 & $1.34 \pm 0.01$ & 4.9 & 0.80 & $2.1 \pm 0.1$ & $-53.5 \pm 5.5$ & & $9.4_{-0.1}^{+0.4}$ & - & - & $87_{-9}^{+1}$ \\
\hline VLA 2.24 & - & 711.0201 & -457.901 & $198.45 \pm 0.20$ & 8.7 & 0.51 & $0.7 \pm 0.1$ & $-72.0 \pm 6.2$ & & $9.0_{-0.3}^{+0.5}$ & 6.1 & $160 \pm 24$ & $66_{-42}^{+11}$ \\
\hline VLA 2.25 & - & 713.0410 & -466.595 & $16.74 \pm 0.02$ & 13.7 & 0.60 & $0.8 \pm 0.1$ & $-44.5 \pm 2.1$ & & $8.9_{-0.1}^{+0.5}$ & - & - & $80_{-38}^{+10}$ \\
\hline VLA 2.26 & - & 716.0303 & -475.323 & $53.72 \pm 0.06$ & -7.7 & 0.68 & $1.2 \pm 0.5$ & $-89.6 \pm 61.8$ & $3.0_{-0.3}^{+0.3}$ & & 2.1 & $79 \pm 18$ & $77_{-38}^{+13}$ \\
\hline VLA 2.27 & - & 777.7941 & -447.278 & $0.67 \pm 0.06$ & -7.7 & 0.66 & $6.1 \pm 0.1$ & $-87.7 \pm 6.5$ & $0.7_{-0.1}^{+1.8}$ & $10.7_{-1.7}^{+0.1}$ & - & - & $71_{-3}^{+13}$ \\
\hline
\end{tabular}

Notes. ${ }^{(a)}$ The water masers associated with VLA 1 are divided in three groups according to their positions (see Sect. 4.1 ). ${ }^{(b)}$ The best-fitting results obtained by using a model based on the radiative transfer theory of water masers (Vlemmings et al. 2006, 2010) for $\Gamma+\Gamma_{v}=1$. The errors were determined by analysing the full probability distribution function. For $T \sim 400 \mathrm{~K}\left(\Gamma_{v}=2\right)$ and $T \sim 2500 \mathrm{~K}\left(\Gamma_{v}=13\right) T_{\mathrm{b}} \Delta \Omega$ has to be adjusted by adding +0.48 and +1.15 , respectively (Nedoluha $\&$ Watson 1992; Anderson $\&$ Watson 1993). ${ }^{(c)}$ The angle between the magnetic field and the maser propagation direction is determined by using the observed $P_{1}$ and the fitted emerging brightness temperature.The errors were determined by analysing the full probability distribution function. ${ }^{(d)}$ Because of the degree of the saturation of these water masers $T_{\mathrm{b}} \Delta \Omega$ is underestimated, $\Delta V_{\mathrm{i}}$ and $\theta$ are overestimated. ${ }^{(e)}$ The constraint fit did not allow us to evaluate the error bars properly. ${ }^{(f)}$ In the fitting model we include the values for $T_{\mathrm{b}} \Delta \Omega$ and $\Delta V_{\mathrm{i}}$ of the closest features. 

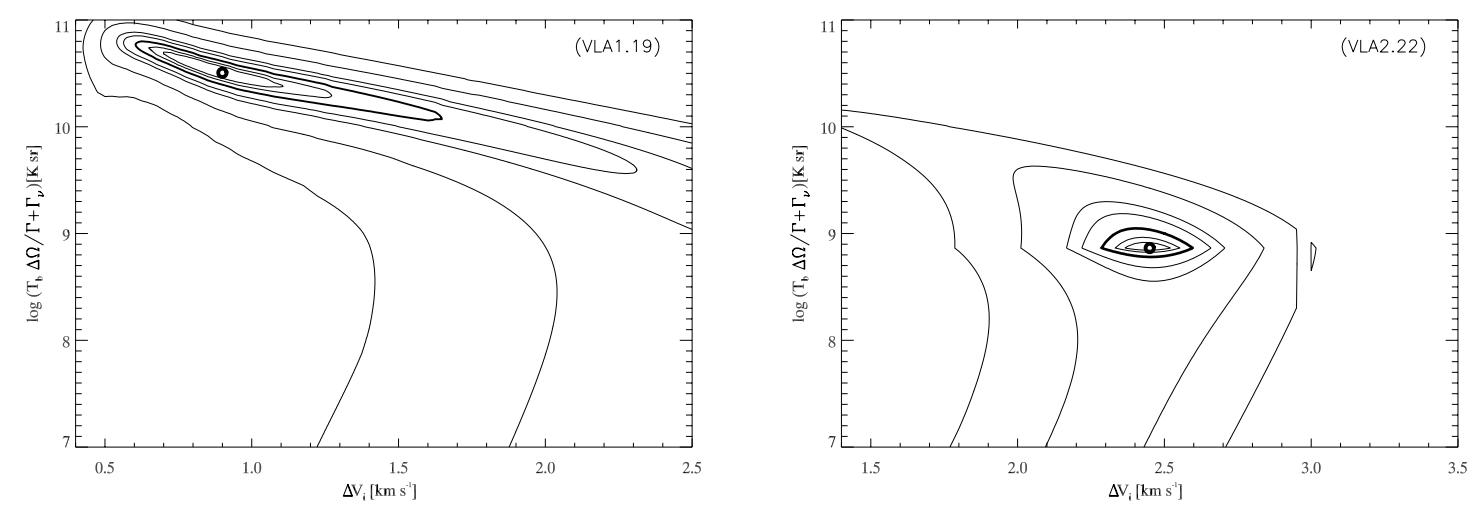

Fig. 1. Results of the full radiative transfer $\chi^{2}$-model fits, for the maser that shows the highest linear polarization fraction (VLA1.19) and for the maser that shows the highest $P_{\mathrm{V}}$ around VLA2 (VLA2.22). The fits yield the emerging maser brightness temperature $T_{\mathrm{b}} \Delta \Omega$ and the intrinsic maser linewidth $\Delta V_{\mathrm{i}}$. Contours indicate the significance intervals $\Delta \chi^{2}=0.25,0.5,1,2,3$, , with the thick solid contours indicating $1 \sigma$ and $3 \sigma$ areas.

which is able to search for maser spots, velocity channel by velocity channel, with a signal-to-noise ratio greater than a given value (in our case, an 8-sigma limit was used); 2) we fitted the identified maser spots using the AIPS task IMFIT; and 3) we identified a maser feature, when three or more maser spots coincided spatially (within a box of 2 by 2 pixels) and each of them appeared in consecutive velocity channels. Table 1 contains the positions, velocities and peak flux densities of all the maser features found in the region.

In the first part of the process and after evaluating the global noise level of the channel map, the code searches for maser spots in the velocity map, excluding the edges of the channel map. When strong maser spots are found (for instance, with a peak flux density higher than $2 \mathrm{Jy}_{\text {beam }}{ }^{-1}$ ), the local rms around these strong maser spots (within a predefined box, for instance, 400 by 400 pixels) is estimated, as well as the rms outside the boxes. The code identifies a maser spot when the SNR of the candidate (using the local rms) is greater than a predefined value. In the case of $\mathrm{W} 75 \mathrm{~N}$, we have adopted a lower limit of 8-sigma. The code produces a table with the spatial location and intensity of all the identified maser spots. This procedure is repeated for each velocity channel included in the input file that is used by the code. The code also produces a script for each velocity channel that can be run inside AIPS in order to produce Gaussian fits of all the identified maser spots. After running all the AIPS scripts, a table with all the important parameters (e.g., spatial resolution, velocity, intensity) of each maser spot is produced. We then use the output table to search for maser features that fulfill the criteria described above.

\subsection{Polarization fitting}

From the maser theory we know that the fractional linear polarization $P_{1}$ of the $\mathrm{H}_{2} \mathrm{O}$ maser emission depends on the degree of its saturation and the angle $(\theta)$ between the maser propagation direction and the magnetic field (e.g., Goldreich et al. 1973; Nedoluha \& Watson 1992). Even the relation between the measured polarization angle $\chi$ and the magnetic field angle on the sky $\left(\phi_{B}\right)$ depends on $\theta$, with the linear polarization vector perpendicular to the field for $\theta>\theta_{\text {crit }} \sim 55^{\circ}$ (Goldreich et al. 1973), where $\theta_{\text {crit }}$ is the so-called Van Vleck angle. It is therefore important to evaluate $\theta$ for every $\mathrm{H}_{2} \mathrm{O}$ maser that shows linear polarization emission. In order to obtain the best values for $\theta$, we needed to determine the emerging brightness temperature $\left(T_{\mathrm{b}} \Delta \Omega\right)$ and the intrinsic thermal linewidth $\left(\Delta V_{\mathrm{i}}\right)$, which is the full width half-maximum (FWHM) of the Maxwellian distribution of particle velocities, of the masers with high precision. This could be done by fitting the water maser emissions with the full radiative transfer method described by Vlemmings et al. (2006, 2010), who successfully used it for water and methanol masers in Cepheus A. These radiative transfer methods are based on the models for water masers of Nedoluha \& Watson (1992), for which the shapes of the total intensity, linear polarization, and circular polarization spectra depend on $T_{\mathrm{b}} \Delta \Omega$ and $\Delta V_{\mathrm{i}}$. They also found that the emerging brightness temperature scaled linearly with $\left(\Gamma+\Gamma_{v}\right)$, which are the maser decay rate $\Gamma$ and crossrelaxation rate $\Gamma_{v}$. Since for the $22 \mathrm{GHz} \mathrm{H} \mathrm{H}_{2} \mathrm{O}$ masers $\Gamma \lesssim 1 \mathrm{~s}^{-1}$ and $\Gamma_{v}$ depends on the gas temperature, we include in our fit a value $\left(\Gamma+\Gamma_{v}\right)=1 \mathrm{~s}^{-1}$, so that we could adjust the fitted $T_{\mathrm{b}} \Delta \Omega$ values by simply scaling according to $\left(\Gamma+\Gamma_{v}\right)$. Note that $\Delta V_{\mathrm{i}}$ and $\theta$ do not need to be adjusted.

We model the observed linear polarized and total intensity maser spectra by gridding the intrinsic thermal linewidth $\Delta V_{i}$ between 0.4 and $3.5 \mathrm{~km} \mathrm{~s}^{-1}$, in steps of $0.025 \mathrm{~km} \mathrm{~s}^{-1}$, using a least square fitting routine $\left(\chi^{2}\right.$-model) with an upper limit of the brightness temperature $T_{\mathrm{b}} \Delta \Omega=10^{11} \mathrm{~K}$ sr. In Fig. 1 two examples are shown of the results obtained by fitting the water maser emissions with the full radiative transfer method code. From these first fits, we can then obtain the angles $\theta$ by considering the relation between $P_{1}$ and $\theta$ (see Vlemmings et al. 2010, for more details). The best values for $T_{\mathrm{b}} \Delta \Omega$ and $\Delta V_{\mathrm{i}}$ are then included in the full radiative transfer code to produce the $I$ and $V$ models that were used for fitting the total intensity and circular polarized spectra of the $\mathrm{H}_{2} \mathrm{O}$ masers extracted from the highresolution $I$ and $V$ cubes. The magnetic field strength along the line of sight is evaluated by using the equation

$$
B_{\|}=B \cos \theta=\frac{P_{V} \Delta v_{L}}{2 \cdot A_{F-F^{\prime}}},
$$

where $\Delta v_{L}$ is the FWHM of the total intensity spectrum, the $A_{\mathrm{F}-\mathrm{F}^{\prime}}$ coefficient, which depends on $T_{\mathrm{b}} \Delta \Omega$. The circular polarization fraction $\left(P_{V}\right)$ are obtained from the $I$ and $V$ models.

Another important aspect that must be considered in the analysis of the water masers is the degree of their saturation, which influences $P_{1}$. We are able to estimate it by considering the ratio between maser rate of stimulated emission $(R)$ and the sum of maser decay rate and the cross-relaxation rate $\left(\Gamma+\Gamma_{v}\right)$.

The stimulated emission rate is given by

$$
R \simeq \frac{A k_{\mathrm{B}} T_{\mathrm{b}} \Delta \Omega}{4 \pi h v}
$$



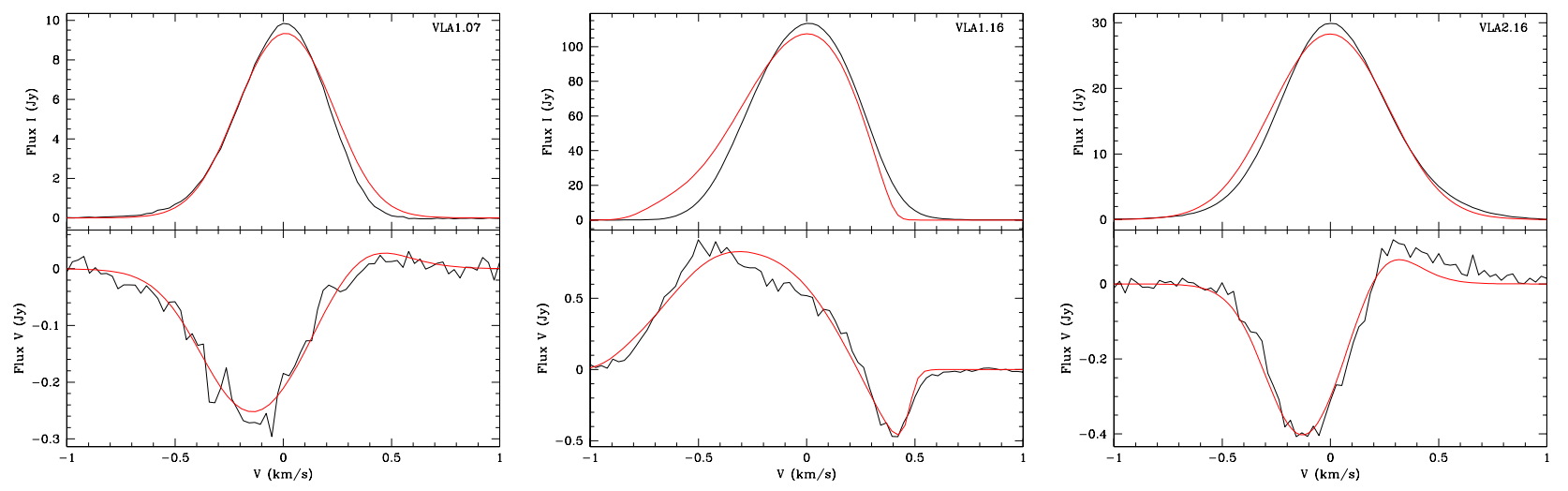

Fig. 2. Total intensity and circular polarization spectrum for the three maser features VLA1.07, VLA1.16, and VLA2.16. The thick red lines are the best-fit models of $I$ and $V$ emission obtained using the radiative transfer method (see Sect. 3.2). All maser features were centered to zero velocity.

where $A=2 \times 10^{-9} \mathrm{~s}^{-1}$ is the $22-\mathrm{GHz} \mathrm{H}_{2} \mathrm{O}$ maser spontaneous rate (Goldreich \& Keeley 1972), $k_{\mathrm{B}}$ and $h$ are the Boltzmann and Planck constants, respectively, $v$ the maser frequency and $T_{\mathrm{b}} \Delta \Omega$ the emerging brightness temperature adjusted at the right $\Gamma+\Gamma_{v}$ value. The masers are unsaturated when $R /\left(\Gamma+\Gamma_{v}\right)<1$, in the onset of saturation when $R /\left(\Gamma+\Gamma_{v}\right) \geq 1$, and fully saturated when $R /\left(\Gamma+\Gamma_{v}\right) \approx 100$ (Vlemmings et al. 2006). Moreover, when the saturation sets in, the maser lines start to broaden again, and this means that the observed linewidth $\left(\Delta v_{\mathrm{L}}\right)$ becomes as large as $\Delta V_{\mathrm{i}}$ or larger depending on the degree of the saturation.

Although the full radiative transfer method is based on a model for general water masers, it should be noted that, when this model is applied to saturated masers, it is impossible to properly disentangle the values of $T_{\mathrm{b}} \Delta \Omega$ and $\Delta V_{\mathrm{i}}$. In this case the method only provides a lower limit for $T_{\mathrm{b}} \Delta \Omega$ and an upper limit for $\Delta V_{\mathrm{i}}$. For high maser brightness temperatures, i.e. greater than $10^{9} \mathrm{~cm}^{-3} \mathrm{~K} \mathrm{sr}$ (Nedoluha \& Watson 1992), the $\cos \theta$ dependence of Eq. (1) breaks down thereby introducing a more complex dependence on $\theta$. As a result, the angle $\theta$ obtained from the fit is overestimated and, in particular, it could also reach values of $90^{\circ}$ or greater. Consequently, the values of $T_{\mathrm{b}} \Delta \Omega, \Delta V_{\mathrm{i}}$ and $\theta$ obtained for saturated masers could not be taken into account in the analysis of the region.

\section{Results}

\subsection{Maser distribution}

In Fig. 3 we show the distribution of the $12422-\mathrm{GHz}$ water maser features detected with the VLBA. No water maser emission with a peak flux density less than $80 \mathrm{mJybeam}^{-1}$ is detected. All $\mathrm{H}_{2} \mathrm{O}$ masers are detected around the radio sources VLA $1(29 \%, 36 / 124)$ and VLA $2(71 \%, 88 / 124)$.

The water masers associated with VLA 2, which have local standard of rest velocities $\left(V_{\mathrm{LSR}}\right)$ between -7.7 and $13.7 \mathrm{~km} \mathrm{~s}^{-1}$, are distributed elliptically around its $1.3 \mathrm{~cm}$ continuum peak. In Fig. 4 we report two ellipses obtained by fitting the positions of the water masers detected by T03 (called here ellipse 1) and in the present paper (ellipse 2), their parameters are reported in Table 2. The major axis of ellipse 2 is 64 mas larger than that of ellipse 1 , the minor axes are also in the same ratio. This indicates an expansion velocity of $\sim 4.8 \mathrm{mas} / \mathrm{yr}$ in all directions, corresponding to $\sim 46 \mathrm{~km} \mathrm{~s}^{-1}$ (at $2 \mathrm{kpc}$ ). This value is consistent with the proper motion of the water masers as reported by T03. Since the expansion velocity is symmetric in all directions, we aligned the centers of the two ellipses with the position of the $1.3 \mathrm{~cm}$ continuum peak obtained from a Gaussian fit. Consequently we were able to overlay all water masers of the region on the continuum VLA map with an accuracy of approximately 10 mas. In this way we could also compare the positions of the masers around VLA 1 at the two different epochs. If we look at the northern masers around VLA2, we see that the masers detected by T03 are better aligned with the ellipse 1 than the masers detected by us with ellipse 2 (Fig. 4, right panel), indicating that those masers are also moving northeastward. This movement might be due to the formation of a jet (see Fig. 3).

The water masers associated with VLA 1 can be divided into three groups, one composed of the northern masers (VLA 1A), one of the masers closest to the central protostar (VLA 1B, see Fig. 5 right panel) and the third one of the southern masers (VLA 1C). The east-northern masers of the VLA 1A group did not show emission during the previous observation of epoch 1999 (T03). All VLA 1A masers $\left(V_{\mathrm{LSR}} \simeq 19-25 \mathrm{~km} \mathrm{~s}^{-1}\right)$ are well-aligned with the direction of the thermal radio jet detected by Torrelles et al. (1997) and the methanol masers detected by S09. The VLA 1B and VLA 1C groups show a distribution quite similar to that shown on April 2nd 1999 (T03). For these two groups T03 reported a proper motion of about $\sim 2 \mathrm{mas} / \mathrm{yr}\left(\sim 19 \mathrm{~km} \mathrm{~s}^{-1}\right)$. Although we have detected several water masers close to those detected by T03, it is very difficult to estimate the proper motion between the two epochs. In fact, as is shown on the right panel of Fig. 5, it is difficult to identify a maser that arises in both epochs (1999 and 2005). The LSR radial velocities of VLA 1 are between 9 and $25 \mathrm{~km} \mathrm{~s}^{-1}$, which are about 3-4 times higher than those of methanol masers (S09) and greater than the systemic velocity of the whole region (10 $\mathrm{km} \mathrm{s}^{-1}$, Shepherd et al. 2003).

\subsection{Linear and circular polarization}

Linear polarization was detected in 50 masers (Table 1). In particular, about $70 \%$ of the $\mathrm{H}_{2} \mathrm{O}$ masers associated with VLA 1 show linear polarization emission. The corresponding linear polarization vectors are shown in Figs. 4 and 5 (left panel). The highest fractional linear polarization was detected in water masers related to VLA $1\left(P_{1} \sim 26 \%\right)$. Eight masers associated with VLA 1 have high linear polarizations, $P_{1}$ between $10 \%$ and $26 \%$, so that the median value of linear polarizations for the masers in VLA 1 is $\sim 7 \%$. In contrast, the maximum $P_{1}$ for the masers associated with VLA 2 is $6 \%$, and the median value is much lower at $1.5 \%$. Circular polarization was detected in 20 masers, equally distributed between VLA 1 and VLA 2. 


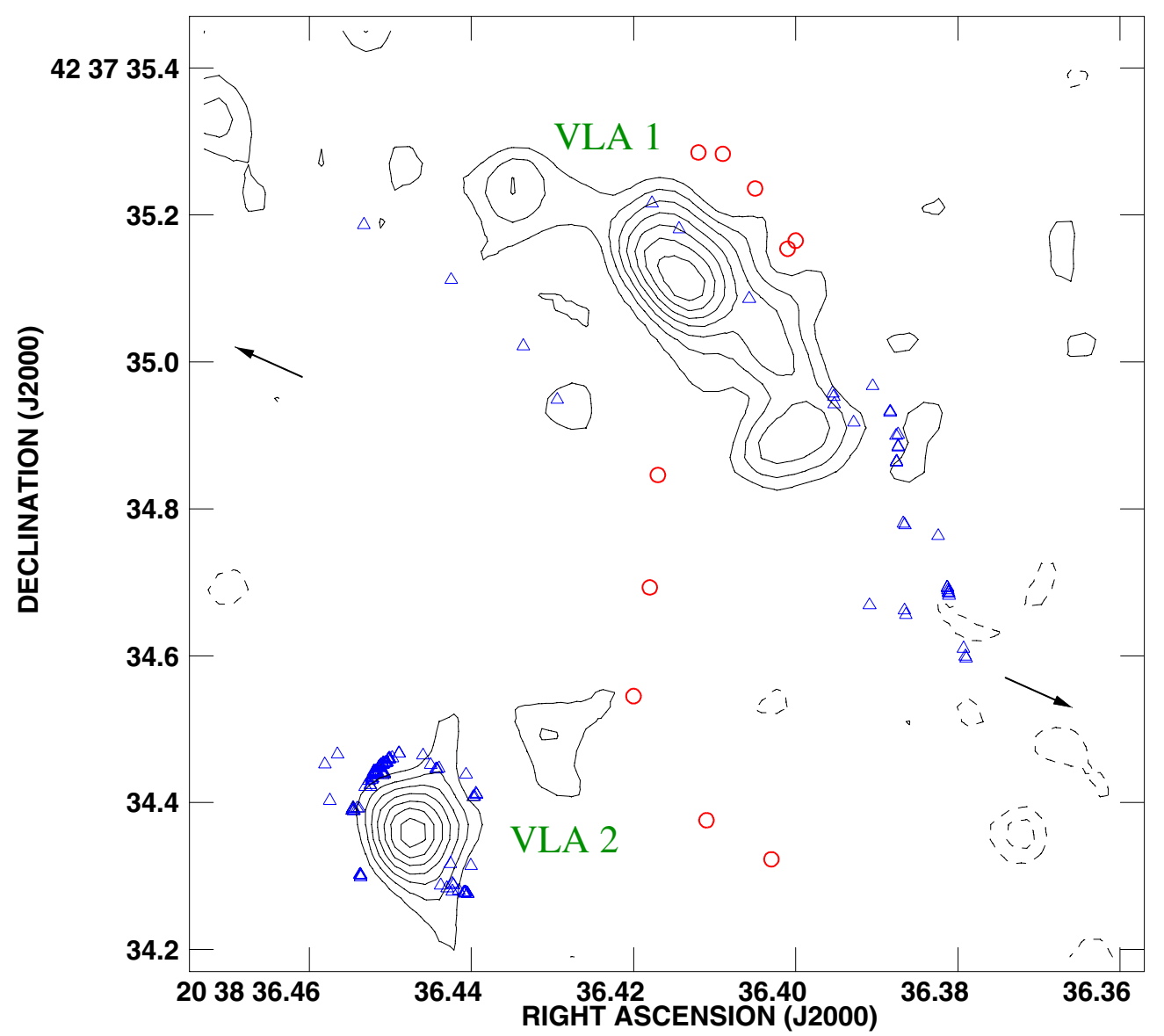

Fig. 3. Positions of water and methanol masers superimposed on the $1.3 \mathrm{~cm}$ continuum contour map of the VLA1 thermal jet and VLA 2 (Torrelles et al. 1997). Contours are $-3,-2,2,3,4,5,6,7,8 \times 0.16 \mathrm{mJy}_{\text {beam }}{ }^{-1}$. Blue triangles indicate the positions of the water maser features detected with the VLBA (this paper). Red circles indicate the position of the methanol masers features detected by S09. The two arrows indicate the direction of the large-scale molecular bipolar outflow $\left(\mathrm{PA}=66^{\circ}\right)$.
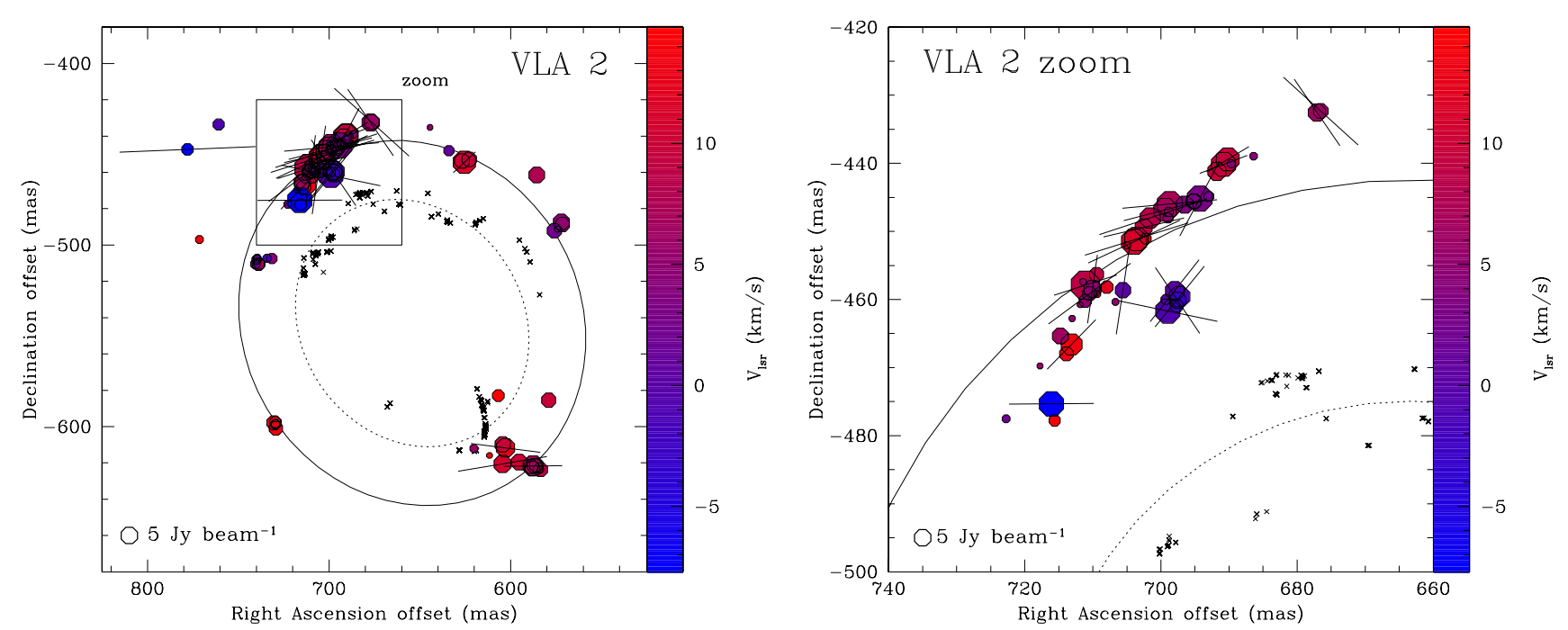

Fig. 4. Left panel: a close-up view of the $\mathrm{H}_{2} \mathrm{O}$ maser features around the radio source VLA 2. Right panel: a zoom-in view of the boxed region of the left panel. The octagonal symbols are the identified maser features in present work scaled logarithmically according to their peak flux density. The maser LSR radial velocity is indicated by color. A $5 \mathrm{Jy} \mathrm{beam}^{-1}$ symbol is plotted for illustration in both panel. The linear polarization vectors, scaled logarithmically according to polarization fraction $P_{1}$ (in Table 1), are overplotted. Two ellipses are also drawn in both panels. They are the results of the best fit of the water masers (crosses) detected by T03 (dotted ellipse, ellipse 1; epoch 1999) and of those detected in present work (solid ellipse, ellipse 2; epoch 2005). Their parameters are listed in Table 4 . The synthesized beam is 2.0 mas $\times 0.7$ mas. 

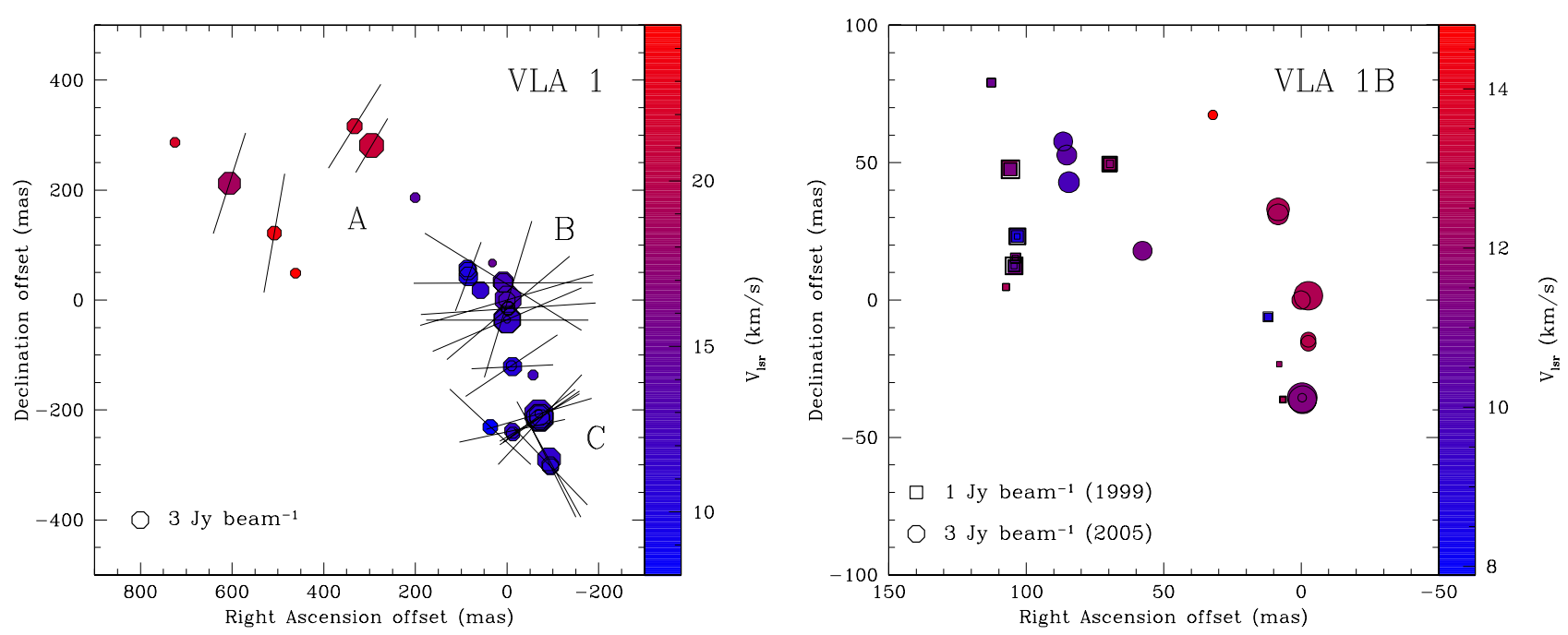

Fig. 5. Left panel: a close-up view of the $\mathrm{H}_{2} \mathrm{O}$ maser features (octagon) around the radio source VLA 1. Right panel: a zoom-in view of the water masers group VLA 1B, here are also reported the water masers (square) detected by T03. The octagonal and the square symbols are scaled logarithmically according to their peak flux density. The maser LSR radial velocity is indicated by color. The systemic LSR radial velocity of W75N massive star-forming region is $10 \mathrm{~km} \mathrm{~s}^{-1}$ (Shepherd et al. 2003). Symbols for $3 \mathrm{Jy} \mathrm{beam}^{-1}$ and for $1 \mathrm{Jy} \mathrm{beam}^{-1}$ are shown in the lower left corner of the panels. The linear polarization vectors, scaled logarithmically according to polarization fraction $P_{1}$ (in Table 1), are overplotted on the left panel. The synthesized beam is 2.0 mas $\times 0.7$ mas.

Table 2. Number, epoch, semi-major axis, semi-minor axis, and inclination from the elliptical fit of the water maser associated with VLA2 in the two different epochs.

\begin{tabular}{cccccc}
\hline \hline Ellipse & Epoch & $\begin{array}{c}a \\
(\mathrm{mas})\end{array}$ & $\begin{array}{c}b \\
(\mathrm{mas})\end{array}$ & $\begin{array}{c}\theta \\
\left({ }^{\circ}\right)\end{array}$ & Ref. \\
\hline 1 & $2-$ Apr.-1999 & 71.0 & 61.1 & 123.5 & T03 \\
2 & 21-Nov.-2005 & 103.1 & 92.9 & 120.5 & this work \\
\hline
\end{tabular}

The rms weighted linear polarization angles of the water masers around VLA 1 and VLA 2 are $\left\langle\chi_{1}\right\rangle \approx-67^{\circ} \pm 40^{\circ}$ and $\left\langle\chi_{2}\right\rangle \approx-72^{\circ} \pm 32^{\circ}$, respectively. Since the water masers of group VLA 1A show ordered linear polarization vectors, it is worthwhile evaluating its weighted polarization angles, which is $\left\langle\chi_{1 \mathrm{~A}}\right\rangle=-20^{\circ} \pm 9^{\circ}$.

We were able to fit 42 masers with the method described in Sect. 3.2, and the results are given in columns 10 and 11 of Table 1, and two examples (i.e., VLA1.19, VLA2.22) are reported in Fig. 1. Although the emerging brightness temperatures for VLA 1 and VLA 2 are both in the range $10^{8} \mathrm{~K} \mathrm{sr}<$ $\mathrm{T}_{\mathrm{b}} \Delta \Omega<10^{10} \mathrm{~K} \mathrm{sr}$, their weighted intrinsic maser linewidths are very different. They are $\left\langle\Delta V_{\mathrm{i}}\right\rangle_{\mathrm{VLA} 1}=1.0_{-0.2}^{+0.8} \mathrm{~km} \mathrm{~s}^{-1}$ and $\left\langle\Delta V_{\mathrm{i}}\right\rangle_{\mathrm{VLA} 2}=2.5_{-0.4}^{+0.6} \mathrm{~km} \mathrm{~s}^{-1}$, respectively.

Finally, considering the emerging brightness temperature and the observed $P_{1}$ we determined $\theta$ for those masers which we were able to fit for $\Delta V_{\mathrm{i}}$ and $T_{\mathrm{b}} \Delta \Omega$. The results are given in Col. 14 of Table 1 with the errors determined by analyzing the full probability distribution functions. Eight of the $\mathrm{H}_{2} \mathrm{O}$ masers around the radio source VLA 1 (from VLA1.12 to VLA1.19, which are the closest features to the protostar) show values for $\theta$ equal to $90^{\circ}$. This suggests that, from the theory of the Zeeman effect, we are observing perpendicular to the magnetic field. Consequently, we should not be able to detect circular polarization from these masers. However, since we detected circular polarization in three of them (VLA1.16, VLA1.17, and VLA1.19) the measurements of $\theta$ must be affected by the degree of their saturation, as discussed in Sect. 3.2. By excluding these 8 masers, we evaluated $\langle\theta\rangle_{\mathrm{VLA} 1}=83^{\circ} \pm 7^{\circ}$ and $\langle\theta\rangle_{\mathrm{VLA} 2}=$ $85^{\circ} \pm 6^{\circ}$, which imply that the magnetic fields are close to the plane of the sky.

In Col. 13 of Table 1 we report the magnetic field strength along the line of sight obtained by fitting the high-resolution spectra of $I$ and $V$ (see Sect. 3.2). The masers VLA1.21, VLA2.01, and VLA2.02 do not show any linear polarization emission, but they do show circular polarization emission. Consequently we could not get $T_{\mathrm{b}} \Delta \Omega$ and $\Delta V_{\mathrm{i}}$ as done for the other masers that showed linear polarization emission, and to measure the magnetic field strength, we used the $T_{\mathrm{b}} \Delta \Omega$ and $\Delta V_{\mathrm{i}}$ values of the closest masers. In Table 1 the circular polarization fraction is also reported (Col. 12).

\section{Discussion}

The water masers detected using the VLBA are related to two different radio continuum sources of $\mathrm{W} 75 \mathrm{~N}(\mathrm{~B}), \mathrm{VLA} 1$ and VLA 2, which are thought to be in two different evolutionary stages. In the next sections, we discuss their nature as two independent groups, their polarization and finally the type of shocks that give rise to them.

\section{1. $\mathrm{H}_{2} \mathrm{O}$ maser properties}

As suggested by the difference between their intrinsic thermal linewidths, which is $1.5 \mathrm{~km} \mathrm{~s}^{-1}$, the masers around VLA 1 and VLA 2 appear to arise under two different physical conditions. Using

$$
\Delta V_{\mathrm{i}} \approx 0.5 \times(T / 100)^{\frac{1}{2}},
$$

we estimate the gas temperatures in the $\mathrm{H}_{2} \mathrm{O}$ masing regions around VLA 1 and VLA 2 , and find them to be $T_{\mathrm{VLA} 1} \sim$ $400 \mathrm{~K}$ and $T_{\mathrm{VLA} 2} \sim 2500 \mathrm{~K}$, respectively. The intrinsic thermal linewidths of the VLA 2 masers are quite similar to each other, while the three groups of masers around VLA 1 show a difference in their $\Delta V_{\mathrm{i}}$. In particular, we find that the maser 
groups VLA 1A and $\mathrm{C}$ have similar intrinsic thermal linewidths $\left(\left\langle\Delta V_{\mathrm{i}}\right\rangle=1.0 \mathrm{~km} \mathrm{~s}^{-1}\right)$ while the group VLA 1B has lower values $\left(\left\langle\Delta V_{\mathrm{i}}\right\rangle=0.7 \mathrm{~km} \mathrm{~s}^{-1}\right)$. This can come from the degree of their saturation that we can evaluate by considering Eq. (2).

Before using Eq. (2) we have to adjust the emerging brightness temperature values as explained in Sect. 3.2. At a temperature of $\sim 400 \mathrm{~K}$, for which $\Gamma+\Gamma_{v}=3 \mathrm{~s}^{-1}$ (Nedoluha \& Watson 1992), the weighted emerging brightness temperature of VLA $1 \mathrm{~B}$ is $\left\langle T_{\mathrm{b}} \Delta \Omega\right\rangle>10^{11} \mathrm{~K} \mathrm{sr}$, which is close to the limit of full saturation, and for VLA $1 \mathrm{~A}$ and $\mathrm{C}$ is $\left\langle T_{\mathrm{b}} \Delta \Omega\right\rangle=10^{10} \mathrm{~K}$ sr. From Eq. (2) the masers of VLA $1 \mathrm{~A}$ and VLA $1 \mathrm{C}$ are thus completely unsaturated $\left(R /\left(\Gamma+\Gamma_{v}\right)=0.5\right)$, while VLA 1B masers are saturated $\left(R /\left(\Gamma+\Gamma_{v}\right)>5\right)$, even if the real degree of their saturation is unknown because of underestimating $T_{\mathrm{b}} \Delta \Omega$. The average observed linewidth $\left(\Delta v_{\mathrm{L}}\right)$ of VLA 1B group, which is about $0.5 \mathrm{~km} \mathrm{~s}^{-1}$, gives us another indication of the saturation state of their $\mathrm{H}_{2} \mathrm{O}$ masers. As reported in Sect. 3.2, the intrinsic thermal linewidths for the saturated masers are overestimated so $\Delta v_{\mathrm{L}}$ might be larger or at least equal to $\Delta V_{\mathrm{i}}$ implying that the maser lines are rebroadened as expected in the saturated maser lines. As the VLA 1B masers are saturated, they have not been taken into account in our conclusions. For the other masers associated with VLA 1, we observed linewidths well below their intrinsic thermal linewidth, and this confirms that these masers are unsaturated. The masers associated with VLA 2 are unsaturated; in fact, for $T \sim 2500 \mathrm{~K}\left(\Gamma+\Gamma_{v}=14 \mathrm{~s}^{-1}\right.$, Anderson \& Watson 1993), we get ratios of $R /\left(\Gamma+\Gamma_{v}\right)<0.5$.

From the measurements of the maser flux densities $(S(v))$ and feature angular sizes $(\Sigma)$ we can estimate the brightness temperature by the equation reported in S09,

$$
\frac{T_{\mathrm{b}}}{[\mathrm{K}]}=\frac{S(v)}{[\mathrm{Jy}]} \cdot\left(\frac{\Sigma^{2}}{\left[\mathrm{mas}^{2}\right]}\right)^{-1} \cdot \xi_{\mathrm{H}_{2} \mathrm{O}},
$$

where $\xi_{\mathrm{H}_{2} \mathrm{O}}=1.24 \times 10^{9} \mathrm{mas}^{2} \mathrm{Jy}^{-1} \mathrm{~K}$ is a constant factor which includes all constant values, such as the Boltzmann constant, the wavelength, and the proportionality factor obtained for a Gaussian shape by Burns et al. (1979). Comparing $T_{\mathrm{b}}$ with the emerging brightness temperatures obtained from the model, we can easily estimate $\Delta \Omega$. The Gaussian fit of the masers give a size of 0.4 mas for the water masers related to VLA 1 , which indicates that they are unresolved, while a size of 0.5 for the masers associated with VLA 2, which indicates that they are marginally resolved. The brightness temperatures of the brightest masers in both groups are $T_{\mathrm{b}, \mathrm{VLA} 1.08}>1.23 \times 10^{12} \mathrm{~K}$ and $T_{\mathrm{b}, \mathrm{VLA} 2.24} \approx 10^{12} \mathrm{~K}$, for which we find $\Delta \Omega_{\mathrm{VLA} 1.08}<10^{-2} \mathrm{sr}$ and $\Delta \Omega_{\mathrm{VLA} 2.24} \approx 10^{-2} \mathrm{sr}$ for $T=400 \mathrm{~K}$ and $2500 \mathrm{~K}$, respectively. Considering all the features in Table 1, the maser beaming of the two groups are $\Delta \Omega_{\mathrm{VLA} 1} \lesssim 10^{-2} \mathrm{sr}\left(\Gamma+\Gamma_{v}=2 \mathrm{~s}^{-1}\right)$ and $\Delta \Omega_{\mathrm{VLA} 2} \approx 10^{-2} \mathrm{sr}\left(\Gamma+\Gamma_{v}=14 \mathrm{~s}^{-1}\right)$. In a tubular geometry $\Delta \Omega \approx(d / l)^{2}$, where $d$ and $l$ are the transverse size and length of the tube respectively. Assuming $d$ approximately the size of the maser features, the maser lengths are all in the range $10^{12} \mathrm{~cm} \lesssim l \lesssim 10^{14} \mathrm{~cm}$.

\subsection{Magnetic field in $W 75 N(B)$}

\subsubsection{Magnetic field strength}

The magnetic field strength along the line of sight was determined from circular polarization measurements for 20 water masers. We detected significant $(\geq 3 \sigma)$ magnetic fields toward 10 masers in VLA 1 and 7 masers in VLA 2 . The detected fields along the line of sight $\left(B_{\|}\right)$range from $-400 \mathrm{mG}$ to $+600 \mathrm{mG}$ for the $\mathrm{H}_{2} \mathrm{O}$ masers around VLA 1 and from $-200 \mathrm{mG}$ to $1000 \mathrm{mG}$ for those associated with VLA 2 (see Table 1). The wide range obtained for $B_{\|}^{\mathrm{VLA} 1}$ and $B_{\|}^{\mathrm{VLA} 2}$ is not anomalous, and a similar range was reported for Cepheus A (Vlemmings et al. 2006). The changing in the sign indicates the reversal of the magnetic field (negative towards the observer, positive away from the observer) as already observed in other sources (e.g., Vlemmings et al. 2006). Since the $\theta$ values are close to $90^{\circ}$, i.e. close to the plane of the sky, a slight difference in these angles can produce an inversion of the sign of the magnetic fields. In the case of VLA 1 , the positive and negative magnetic field strengths measured for VLA 1B and VLA 1C groups indicate a twisted magnetic field, which is not the case for VLA 2. Apart from the $\mathrm{H}_{2} \mathrm{O}$ masers VLA2.01 and VLA2.02, which are located on the right edge of the ellipse 2 and show positive values, the other masers in the upper left part of the ellipse 2 (from VLA2.09 to VLA2.26) present a clear separation between positive and negative magnetic field strengths (see Fig. 4 and Table 1).

Based on these detections the absolute weighted magnetic field strengths along the line of sight, where the weights are $w_{i}=1 / e_{i}^{2}$ and $e_{i}$ is the error of the $i$ th measurement, are $\left\langle\left|B_{\|}^{\mathrm{VLA} 1}\right|\right\rangle=(81 \pm 62) \mathrm{mG}$ and $\left\langle\left|B_{\|}^{\mathrm{VLA} 2}\right|\right\rangle=(145 \pm 110) \mathrm{mG}$. In the case of VLA 1 , it is more correct to determine $\left\langle\left|B_{\|}\right|\right\rangle$for only the unsaturated masers; i.e., $\left\langle\left|B_{\|}^{V L A 1}\right|\right\rangle=(74 \pm 50) \mathrm{mG}$. The large errors are due to the large scatter of the magnetic field strength values, which depend on the projection effects, on the density of each water maser region, and on the local velocities of shocks. Because of this scatter it is difficult to evaluate the true strength of the full magnetic fields of the entire region. To compare the results obtained using different maser species we can estimate them roughly. Since $\langle\theta\rangle_{\mathrm{VLA} 1}=83^{\circ}{ }_{-15^{\circ}}$ and $\langle\theta\rangle_{\mathrm{VLA} 2}=85_{-36^{\circ}}^{\circ}$ the absolute weighted magnetic field strengths are $\left|B_{\mathrm{VLA} 1}\right|=\left\langle\left|B_{\|}^{\mathrm{VLA} 1}\right|\right\rangle / \cos \langle\theta\rangle_{\mathrm{VLA} 1} \sim 700 \mathrm{mG}$ and $\left|B_{\mathrm{VLA} 2}\right| \sim 1700 \mathrm{mG}$, respectively. Such high values are also found by Vlemmings et al. (2006) in Cepheus A. As their shock velocity is $10 \mathrm{~km} \mathrm{~s}^{-1}$, measuring a magnetic field of $600 \mathrm{mG}$, these authors explain this high value with the presence of a nearby magnetic dynamo.

If the magnetic field is important throughout the collapse of a spherical cloud, the cloud forms primarily by flows along field lines, and the conservation of magnetic flux and of the mass imply $B \propto n^{0.47}$ (Crutcher 1999). Although the scaling of the magnetic field in water maser is due to shocks, Vlemmings (2006, 2008) empirically showed that even water masers with number densities up to $10^{11} \mathrm{~cm}^{-3}$ follow this relation, even if in non-masing gas of similar densities the magnetic field strengths are likely lower due to ambipolar diffusion. Hence, from the magnetic field strengths, we can determine the number density of the cloud where the $\mathrm{H}_{2} \mathrm{O}$ masers arise by the relation $B \propto n^{0.47}$. Considering the pre-shock magnetic field $\left(B_{\|}=16 \mathrm{mG}\right)$ and density $\left(n_{\mathrm{H}_{2}}=10^{9} \mathrm{~cm}^{-3}\right)$ obtained by S09 from methanol maser emissions, we have $n_{\mathrm{H}_{2}}^{\mathrm{VLA} 1}=3 \times 10^{10} \mathrm{~cm}^{-3}$ and $n_{\mathrm{H}_{2}}^{\mathrm{VLA} 2}=1 \times 10^{11} \mathrm{~cm}^{-3}$. Similar calculation can be done considering the magnetic field strength obtained during the $\mathrm{OH}$ flare near VLA 2 (Slysh et al. 2010). They measured a magnetic field strength of about $70 \mathrm{mG}$, which is comparable to the total magnetic field strength inferred by $\mathrm{S} 09(B=50 \mathrm{mG})$. Since from the $\mathrm{OH}$ maser observations is generally possible to get only $B$ and not $B_{\|}$and since the flare occurred near VLA 2 , in this case we have to use the value of $\left|B_{\mathrm{VLA} 2}\right|$ instead of the value of $\left\langle\left|B_{\|}^{\mathrm{VLA} 2}\right|\right\rangle$. For an $\mathrm{OH}$ number density of $10^{8} \mathrm{~cm}^{-3}$, we find $n_{\mathrm{H}_{2}}^{\mathrm{VLA} 2} \approx 9 \times 10^{10} \mathrm{~cm}^{-3}$ for the water masers around VLA 2, close 
to what is obtained considering the methanol masers. These results, which are close to the extreme upper limit of $10^{11} \mathrm{~cm}^{-3}$ of the water maser thermalisation (Elitzur et al. 1989), confirm the assumptions on the $\mathrm{CH}_{3} \mathrm{OH}$ and $\mathrm{OH}$ number densities and reinforce the measurements of the magnetic field strength made by S09, Slysh et al. (2010) and in this paper. The three independent magnetic field strength values obtained from three different maser species located at different positions show that the magnetic field in massive star-forming regions can reach strength larger than expected. This large unexpected values of $B$ might be due either to the presence of a magnetic dynamo or to the presence of very high-velocity shocks that strongly compress the gas, and consequently increase the magnetic field strength from 50-70 mG (pre-shock methanol-OH region) to $>1$ Gauss (water maser region). For the last hypothesis we refer the reader to Sect. 5.3.

With regard to the orientation of the magnetic field, it is worthwhile to discuss the influence of the Faraday rotation in our observations.

\subsubsection{Faraday rotation}

The measurements of the linear polarization angle $(\chi)$ might be disturbed by the foreground, ambient, and internal Faraday rotation, which are given by

$$
\Phi\left[^{\circ}\right]=1.35 \times 10^{-15} D[\mathrm{~cm}] n_{\mathrm{e}}\left[\mathrm{cm}^{-3}\right] B_{\|}[\mathrm{mG}] v^{-2}[\mathrm{GHz}],
$$

where $D$ is the length of the path over which the Faraday rotation occurs, $n_{\mathrm{e}}$ and $B_{\|}$are respectively the average electron density and the magnetic field along this path and $v$ the frequency. For the foreground Faraday rotation (i.e. the rotation due to the medium between the source and the observer) $\phi_{\mathrm{f}}$ is 0.4 at $22 \mathrm{GHz}$, assuming the interstellar electron density and the magnetic field are $n_{\mathrm{e}} \approx 0.012 \mathrm{~cm}^{-3}$ and $B_{\|} \approx 2 \mu \mathrm{G}$, respectively.

All water masers are not in the same plane, but they arise in clouds at different depths. This depth is impossible to determine from our observations, so in order to estimate the ambient Faraday rotation (i.e. the rotation due to the ambient medium where masers arise), we assume the maximum projected separation as upper limit of $D$ among masers close to each other with the highest velocity difference, which is $D_{\max } \approx 2 \times 10^{15} \mathrm{~cm}$. For the electron density, we assume the value reported by Fish $\&$ Reid (2006), $n_{\mathrm{e}} \approx 300 \mathrm{~cm}^{-3}$, which can produce a rotation of $90^{\circ}$ along the path amplification of $\mathrm{OH}$ maser around $\mathrm{H}$ II regions. Considering $B_{\|}=16 \mathrm{mG}$ (S09), the ambient Faraday rotation is $\phi_{\mathrm{a}}<26^{\circ}$.

The internal Faraday rotation, which can destroy the linear polarization (Fish \& Reid 2006), depends on the type of shock that pumps the water masers. They can either be a dissociative jump shock (J-shock) or a nondissociative continuous shock (C-shock). In the case of a C-shock, the intrinsic Faraday rotation at $22 \mathrm{GHz}$ can be considered negligible because the ionization state of the gas is controlled by cosmic-ray ionization, which generates electron-molecular ion pairs at a rate of $10^{-17} \mathrm{~s}^{-1}$ (Kaufman \& Neufeld 1996), while we have to roughly estimate its contribution for a J-shock. In this case, the electron density is $n_{\mathrm{e}}=\chi_{\mathrm{e}} n_{\mathrm{H}_{2}}$, where $\chi_{\mathrm{e}}$ is the ionization fraction $\left(\chi_{\mathrm{e}}=10^{-5}-10^{-4}\right.$; Kylafis \& Norman 1987) and $n_{\mathrm{H}_{2}}$ the number density of the $\mathrm{H}_{2} \mathrm{O}$ masers. In the most conservative situation, for which $n_{\mathrm{e}} \sim 10^{4} \mathrm{~cm}^{-3}$, we determine an internal Faraday rotation of tens of degrees, enough to destroy the linear polarization in some cases. This means that either the electron density must be much lower or, more likely, the internal Faraday rotation must be negligible, and consequently the shock must be a C-shock.

\subsubsection{Magnetic field orientation}

Suppose that the water masers in both sources are pumped by $\mathrm{C}$-shocks (see Sect. 5.3), this means that the Faraday rotation is $<26^{\circ}$. Since the $\mathrm{H}_{2} \mathrm{O}$ masers associated with VLA 1 and VLA 2 indicate that there are two different magnetic fields around the two sources, it is worthwhile discussing them separately. All water masers show $\theta>\theta_{\text {crit }} \sim 55^{\circ}$; i.e., the magnetic field direction is perpendicular to the linear polarization vectors and close to the plane of the sky.

The group VLA 1A shows an orientation of the magnetic field $\varphi_{\mathrm{B}}^{\mathrm{VLA} 1 \mathrm{~A}}=70^{\circ} \pm 9^{\circ}$, which is in good agreement with what is reported by $\operatorname{S} 09\left(\varphi_{\mathrm{B}}=73^{\circ} \pm 10^{\circ}\right)$. This also confirms that the magnetic field orientation in this part of the source is close to the direction of the large-scale molecular bipolar outflow $\left(66^{\circ}\right)$ (S09). The other two groups VLA 1B and C show disordered linear polarization vectors, which cannot give information on the orientation of the magnetic field. The different orientation of the linear polarization vectors for these two groups might come from the different depths of the masers, i.e. high values of the ambient Faraday rotation, and therefore we can assume that the masers with angles far from $-20^{\circ}$ are located at higher depths than those with angles close to $-20^{\circ}$ (e.g. VLA1.14).

Although around VLA 2 some $\mathrm{H}_{2} \mathrm{O}$ masers show the $90^{\circ}$ flip of the linear polarization angle (e.g., VLA2.15), which was also observed in some water masers in Cepheus A (Vlemmings et al. 2006), the magnetic field appears to be radial (see Fig. 4). Considering $\left\langle\chi_{2}\right\rangle \approx-72^{\circ}$, the magnetic field orientation is about $18^{\circ}$ that indicates a misalignment with the large-scale molecular bipolar outflow and the magnetic field of VLA 1, even though the inclination of both ellipses $\left(\sim 60^{\circ}\right)$ is close to them.

The different orientation of the magnetic fields of the two sources again implies different local physical environments for VLA 1 and VLA 2, and suggests that VLA 1 is the powering source of the large-scale molecular bipolar outflow, as already indicated by S09.

\subsection{J- or C-shocks?}

As reported in previous papers, the $\mathrm{H}_{2} \mathrm{O}$ masers are thought to be pumped by the transition of shocks generated in the two protostars (e.g., Torrelles et al. 1997). These shocks can be either $\mathrm{C}$-shocks or J-shocks. In a water masing region, C-shocks show $v \lesssim 50 \mathrm{~km} \mathrm{~s}^{-1}$ and a post-shock $T<4000 \mathrm{~K}$ (Kaufman \& Neufeld 1996), while J-shocks have velocities $v \gtrsim 45 \mathrm{~km} \mathrm{~s}^{-1}$ and a post-shock plateau temperature that depends on the pre-shock density, the shock velocity, and the observed linewidth of the water masers (Elitzur et al. 1989). Kylafis \& Norman (1991) investigated the effects of the temperature on their J-shock model up to a temperature of about $1000 \mathrm{~K}$, and they found that the brightness temperature starts to increase more slowly when a temperature $\sim 500 \mathrm{~K}$ is reached.

We first consider VLA 1. Assuming the proper motion of the water masers reported by T03, i.e. $19 \mathrm{~km} \mathrm{~s}^{-1}$, as the velocity of the shock and as pre-shock density, the density of the methanol masers $n_{\mathrm{H}_{2}}=10^{9} \mathrm{~cm}^{-3}(\mathrm{~S} 09)$, the equation reported in Elitzur et al. (1989) for a J-shock

$$
T_{\text {plateau }} \approx 400\left(\frac{n_{0}}{10^{7} \mathrm{~cm}^{-3}} \frac{v_{\mathrm{s}}}{100 \mathrm{~km} \mathrm{~s}^{-1}} \frac{\Delta v^{-1}}{1 \mathrm{~km} \mathrm{~s}^{-1}}\right)^{\frac{2}{9}} \mathrm{~K},
$$

where here $\Delta v_{\mathrm{VLA} 1}=0.5 \mathrm{~km} \mathrm{~s}^{-1}$, gives us a most likely postshock temperature $T_{\text {plateau }}^{\mathrm{VLA} 1} \approx 900 \mathrm{~K}$, which is higher than the temperature measured considering the weighted intrinsic velocity obtained from our fit $\left(T_{\mathrm{VLA} 1} \approx 400 \mathrm{~K}\right)$. Moreover, from the 
model of a C-shock described by Kaufman \& Neufeld (1996), we are able to estimate the velocity of the shock by considering the temperature $T_{\mathrm{VLA} 1}$. The model for a temperature of $400 \mathrm{~K}$ gives $v_{\mathrm{s}}^{\mathrm{VLA} 1} \sim 15 \mathrm{~km} \mathrm{~s}^{-1}$, which is close to the proper motion of the $\mathrm{H}_{2} \mathrm{O}$ masers. Therefore, following our arguments, the shock in VLA 1 is most likely a C-shock.

In the case of VLA2, the plateau temperature due to a $\mathrm{J}$-shock is $T_{\text {plateau }}^{\mathrm{VLA} 2} \approx 1000 \mathrm{~K}$ (where $\Delta v_{\mathrm{VLA} 2}=0.7 \mathrm{~km} \mathrm{~s}^{-1}$, and $\left.v_{\mathrm{VLA} 2}=46 \mathrm{~km} \mathrm{~s}^{-1}\right)$, which is less than the temperature that we measured $\left(T_{\mathrm{VLA} 2} \approx 2500 \mathrm{~K}\right)$. The difference of the two temperatures may be due to the turbulence velocity of the gas, which can be determined by considering $\Delta V_{\mathrm{i}}$. In fact, $\Delta V_{\mathrm{i}}$ is related to the thermal and turbulence velocities by the equation

$$
\Delta V_{\mathrm{i}}=\sqrt{\Delta V_{\mathrm{th}}^{2}+\Delta V_{\mathrm{turb}}^{2}}
$$

and considering Eq. (3) we are able to estimate the turbulence velocity of the gas if a J-shock is present. The thermal velocity, which stems from the warming up of the gas by the compression due to the passage of a shock, for a temperature of $1000 \mathrm{~K}$ is $\Delta V_{\text {th }}^{\mathrm{VLA} 2} \approx 1.6 \mathrm{~km} \mathrm{~s}^{-1}$, so we have $\Delta V_{\text {turb }} \approx 1.9 \mathrm{~km} \mathrm{~s}^{-1}$. If instead we suppose that the shock is a C-shock from the model of Kaufman \& Neufeld (1996) for $T_{\mathrm{VLA} 2} \sim 2500 \mathrm{~K}$, we get $v_{\mathrm{s}}^{\mathrm{VLA} 2} \sim$ $43 \mathrm{~km} \mathrm{~s}^{-1}$, which is close to the expansion velocity that we determined in the present paper $\left(v_{\mathrm{VLA} 2}=46 \mathrm{~km} \mathrm{~s}^{-1}\right)$. Both velocities are also in good agreement with the velocity of $43 \mathrm{~km} \mathrm{~s}^{-1}$ measured by Slysh et al. (2010) for the $\mathrm{OH}$ masers near VLA 2 obtained by multiepoch observations. However, $v_{\mathrm{VLA} 2}$ is close to the limit of $45 \mathrm{~km} \mathrm{~s}^{-1}$, which is considered the limit between the two types of shocks, so we cannot rule one of them out by only considering the velocity. Since we obtain well-ordered high linear polarization fraction and linear polarization vectors, we have to suppose that the Faraday rotation is at most $\sim 20^{\circ}$; otherwise, the vectors no longer appear to be aligned with the large structure (i.e., ellipse 2). This condition is only met if the internal Faraday rotation is small; in other words, if the shock is a C-shock (Kaufman \& Neufeld 1996). Therefore, we strongly suggest that there is a C-shock also in VLA 2 even if a J-shock cannot be completely ruled out.

From the equation

$$
\frac{B_{\mathrm{maser}}}{[\mathrm{mG}]} \approx 80 \sqrt{\frac{n_{\mathrm{H}_{2}}}{\left[10^{8} \mathrm{~cm}^{-3}\right]}} \cdot \frac{v_{\mathrm{s}}}{\left[10 \mathrm{~km} \mathrm{~s}^{-1}\right]},
$$

where $n_{\mathrm{H}_{2}}$ is the pre-shock density and $v_{\mathrm{s}}$ the velocity of the shock, we can verify our assumption about the pre-shock density and the rough estimation of $\left|B_{\mathrm{VLA} 1}\right|$ and $\left|B_{\mathrm{VLA} 2}\right|$. Equation (8) is valid for both types of shocks (Kaufman \& Neufeld 1996). Considering $n_{\mathrm{H}_{2}}=10^{9} \mathrm{~cm}^{-3}$ as before, we get $\left|B_{\mathrm{VLA} 1}\right| \approx 500 \mathrm{mG}$ and $\left|B_{\mathrm{VLA} 2}\right| \approx 1200 \mathrm{mG}$, which are consistent with the rough values reported in Sect. 5.2, making the presence of a magnetic dynamo unnecessary. Finally, from our results we can strongly depict the following scenario for VLA 1: the hot gas in a C-shock pumps the water masers and at the same time the warm dust associated with this shock emits infrared photons, which pumps the methanol masers in the pre-shock region, as already suggested in S09.

\section{Conclusions}

We observed the $22 \mathrm{GHz}$ water masers in full polarization mode in the massive star-forming region $\mathrm{W} 75 \mathrm{~N}$ with the VLBA. We detected 124 water masers around the two radio sources VLA 1 and VLA 2, which appear to be in two different evolutionary stages; i.e., VLA 1 is more evolved and a radio jet has not formed yet in VLA 2. From our observations, we have shown that these two sources separated in the sky by $\sim 1^{\prime \prime}$ (2000 AU) have different local physical environments. In particular, the linear polarization indicates that the magnetic field around VLA 1 is aligned well with the large-scale molecular bipolar outflow, while the magnetic field is well-ordered around the shell-like expanding gas associated with VLA 2. The detection of Zeemansplitting indicates an absolute weighted magnetic field strengths $\left|B_{\mathrm{VLA} 1}\right| \sim 700 \mathrm{mG}$ and $\left|B_{\mathrm{VLA} 2}\right| \sim 1700 \mathrm{mG}$. We were also able to determine the type of shocks that pump the water masers associated with both sources. They both are C-shocks, even though a J-shock cannot completely be ruled out in the case of VLA 2. We suggest VLA 1 as the driving source of the large-scale molecular bipolar outflow.

Acknowledgements. We wish to thank an anonymous referee and the editor for making useful suggestions that have highly improved the paper. G.S. and W.H.T.V. acknowledge support by the Deutsche Forschungsgemeinschaft (DFG) through the Emmy Noether Reseach grant VL 61/3-1. S.C. acknowledges support from CONACYT grant 60581. J.M.T. acknowledges support from MICINN (Spain) AYA2008-06189-C03 (co-funded with FEDER funds) and from Junta de Andalucía (Spain).

\section{References}

Alakov, A. V., Slysh, V. I., Popov, M. V., et al. 2005, Astron. Lett., 31, 375 Anderson, N., \& Watson, W. D. 1993, ApJ, 407, 620

Baart, E. E., Cohen, R. J., Davies, R. D., et al. 1986, MNRAS, 219, 145

Burns, J. O., Owen, F. N., \& Rudinick, L. 1979, AJ, 84, 1683

Carrasco-González, C., Rodríguez, L. F., Torrelles, J. M., et al. 2010, AJ, 139, 2433

Crutcher, R. M. 1999, ApJ, 520, 706

Dickel, J. R., Dickel, H. R., \& Wilson, W. J. 1978, ApJ, 223, 840

Elitzur, M., Hollenbach, D. J., \& McKee, C. F. 1989, ApJ, 346, 983

Fish, V. L., \& Reid, M. J. 2006, ApJS, 164, 99

Fish, V. L., \& Reid, M. J. 2007, ApJ, 656, 952

Girart, J. M., Beltrán, M. T., Zhang, Q., et al. 2009, Science, 324, 1408

Goldreich, P., \& Keeley, D. A. 1972, ApJ, 174, 517

Goldreich, P., Keeley, D. A., \& Kwan, J. Y. 1973, ApJ, 179, 111

Haschick, A. D., Reid, M. J., Burke, B. F., et al. 1981, ApJ, 244, 76

Hunter, T. R., Taylor, G. B., Felli, M., et al. 1994, A\&A, 284, 215

Hutawarakorn, B., \& Cohen, R. J. 1996, ASPC, 97, 532

Hutawarakorn, B., Cohen, R. J., \& Brebner, G. C. 2002, MNRAS, 330, 349

Imai, H., Horiuchi, S., Deguchi, S., \& Kameya, O. 2003, ApJ, 595, 285

Kaufman, M. J., \& Neufeld, D. A. 1996, ApJ, 456, 250

Kemball, A. J., Diamond, P. J., \& Cotton, W. D. 1995, A\&AS, 110, 383

Kylafis, N. D., \& Norman, C. A. 1987, ApJ, 323, 346

Kylafis, N. D., \& Norman, C. A. 1991, ApJ, 373, 525

Leppänen, K., Liljeström, T., \& Diamond, P. 1998, ApJ, 507, 909

Matsumoto, T., \& Tomisaka, K. 2004, ApJ, 616, 266

McKee, C. F., \& Ostriker, E. C. 2007, ARA\&A, 45, 565

Minier, V., Booth, R. S., \& Conway, J. E. 2000, A\&A, 362, 1093

Nedoluha, G. E., \& Watson, W. D. 1992, ApJ, 384, 185

Persi, P., Tapia, M., \& Smith, H. A. 2006, A\&A, 445, 971

Reid, M. J., \& Moran, J. M. 1981, ARA\&A, 19, 231

Sarma, A. P., Troland, T. H., \& Romney, J. D. 2001, ApJ, 554, L217

Shepherd, D. S., Testi, L., \& Stark, D. P. 2003, ApJ, 584, 882

Slysh, V. I., \& Migenes, V. 2006, MNRAS, 369, 1497

Slysh, V. I., Migenes, V., Val'tts, I. E., et al. 2002, ApJ, 564, 317

Slysh, V. I., Alakov, A. V., \& Migenes, V. 2010, MNRAS, 404, 1128

Surcis, G., Vlemmings, W. H. T., Dodson, R., et al. 2009, A\&A, 506, 757 (S09)

Torrelles, J. M., Gómez, J. F., Rodríguez, L. F., et al. 1997, ApJ, 489, 744

Torrelles, J. M., Patel, N. A., Anglada, G., et al. 2003, ApJ, 598, L115 (T03)

Vlemmings, W. H. T. 2008, A\&A, 484, 773

Vlemmings, W. H. T., Diamond, P. J., van Langevelde, H. J., et al. 2006, A\&A, 448,597

Vlemmings, W. H. T., Surcis, G., Torstensson, K. J. E., et al. 2010, MNRAS, 404, 134 